\title{
Applied Mathematical Programming and Modelling 2016
}

\author{
Ronald Hochreiter ${ }^{1, \star}$ \\ ${ }^{1}$ WU Vienna University of Economics and Business, Welthandelsplatz 1, 1020 Vienna, Austria.
}

\begin{abstract}
The 12th International Conference on Applied Mathematical Programming and Modelling (APMOD 2016) was held in Brno, Czech Republic in June 8-10, 2016. In this volume eight research papers are collected which depict the broad range of topics the conference series covers.
\end{abstract}

\section{Introduction}

The 12th International Conference on Applied Mathematical Programming and Modelling (APMOD 2016) was held in Brno, Czech Republic in June 8-10, 2016. APMOD (APplied mathematical programming and MODelling) is a series of conferences which was started in 1991 due to an initiative of Gautam Mitra at Brunel University/West London. This initial conference was then followed by events which were held in Budapest (1993), Brunel (1995), Limassol/Cyprus (1998), Brunel (2000), Varenna/Italy (2002), Brunel (2004), Madrid (2006), Bratislava (2008), Paderborn (2012), and Warwick (2014).

The conference accepts contributions from modeling, solving methods and software as well as applications of optimization. Furthermore, we are welcoming contributions from the field of data science as well as business analytics especially in relation to optimization. Relevant application areas include, but are not restricted to finance, transportation, supply chain planning and management, revenue management, energy and infrastructure. The models may be deterministic or stochastic aspects considering robustness or disruption management are welcome. The methodological focus of the previous conferences has been on mathematical programming including stochastic programming, and more recently also heuristics, metaheuristics and simulation.

In this volume eight research papers are collected which depict the broad range of topics the conference series covers.

In Explaining the Results of an Optimization-Based Decision Support System - A Machine Learning Approach by Michael Morin, Rallou Thomopoulos, Irene Abi-Zeid, Maxime Leger, Francois Grondin, and Martin Pleau the authors present their work and explain the results of a commercial software used for real-time decision support for the flow management of a combined wastewater network. The tool is deployed in many major cities and is used on a daily basis and the results show that their approach is very promising according to reactions from analysts and potential users.

The next paper Multicommodity Flow Problems with Commodity Compatibility Relations by Zhiyuan Lin and Raymond Kwan is concerned with a class of Multicommodity Flow Problems with

^e-mail: ronald.hochreiter@wu.ac.at 
Commodity Compatibility Relations (MCFP-CCR), in which compatibility relations among commodities used at each node are required. This class of problems has application in the Train Unit Scheduling Problem (TUSP), where train units of different traction types may not be coupled with each other to serve the same train trip.

Named Entity Recognition: Resource Constrained Maximum Path by Luigi Di Puglia Pugliese, Elisabetta Fersini, Francesca Guerriero, and Enza Messina considers the open research field of Information Extraction (IE) which relates to Named Entity Recognition (NER). In their work they investigate one of the most promising model for NER based on Conditional Random Fields (CRFs). CRFs are enhanced in a two stages approach to include logic rules in the decision process that can be either extracted from data or defined by domain experts.

In their paper MIP models for the irregular strip packing problem: new symmetry breaking constraints Marcos Okamura Rodrigues, Luiz Henrique Cherri, and Leandro Resende Mundim consider the irregular strip packing problem which minimizes the length used to cut a set of pieces from a board with fixed width. A set of new symmetry breaking constraints are proposed to improve previously proposed models. Their results show the proposed formulation is better than the previous one for most instances.

Jan Navratil presents Aerodynamic shape optimization of an airliner elastic wing and compares with optimization of the same wing assuming rigid structure. A gradient-based optimization approach is applied in connection with adjoint method used for calculation of gradients of aerodynamic forces. The initial wing shape is parameterized by Free Form Deformation technique. The aerodynamics of the elastic wing is calculated using the fluid-structure interaction method which couples computational fluid dynamic solver with linear structural solver.

Emergency logistics is acquiring a crucial role aiming to provide adequate care and support for people affected by catastrophic situations; dealing with a large number of stakeholders, scarcity of resources and high stakes. Considering the importance of balancing resources from different participants to improve operations, Oscar Rodriguez-Espindola, Pavel Albores, and Christopher Brewster introduce a bi-criteria response optimisation model aiming to maximise the service provided to disaster victims while making efficient use of resource in their paper Resource management for flood response in Mexico.

Terminal Control Area Aircraft Scheduling and Trajectory Optimization Approaches by Marcella Sama, Konstantin Palagachev, Andrea D'Ariano, Matthias Gerdts, and Dario Pacciarelli is supporting aviation authorities with optimization methods to better use the available infrastructure and better manage aircraft movements. Their paper deals with the real-time scheduling of take-off and landing aircraft at a busy terminal control area and with the optimization of aircraft trajectories during the landing procedures.

Finally, Christoph Waldhauser and Ronald Hochreiter combine the knowledge of Operations Research and Data Mining and apply this to the field of Political Science in their paper Shaking the trees: Abilities and Capabilities of Regression and Decision Trees for Political Science. They compare the established method of analyzing roll call data using W-NOMINATE scores to a data-driven supervised machine learning method: Regression and Decision Trees (RDTs). Two scenarios are defined, one pertaining to an analytical goal, the other being aimed at predicting unknown voting behavior. 